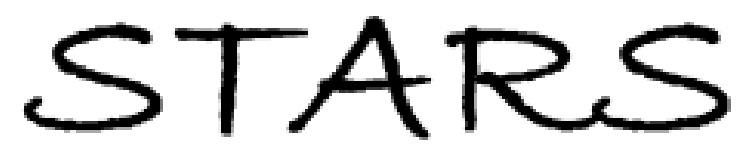

University of Central Florida

STARS

Faculty Bibliography 2000s

Faculty Bibliography

$1-1-2007$

\title{
High-energy two-electron capture with emission of a single photon
}

\author{
E. G. Drukarev
}

A. I. Mikhailov

I. A. Mikhailov

University of Central Florida

W. Scheid

Find similar works at: https://stars.library.ucf.edu/facultybib2000 University of Central Florida Libraries http://library.ucf.edu

This Article is brought to you for free and open access by the Faculty Bibliography at STARS. It has been accepted for inclusion in Faculty Bibliography 2000s by an authorized administrator of STARS. For more information, please contactSTARS@ucf.edu.

\section{Recommended Citation}

Drukarev, E. G.; Mikhailov, A. I.; Mikhailov, I. A.; and Scheid, W., "High-energy two-electron capture with emission of a single photon" (2007). Faculty Bibliography 2000s. 7070.

https://stars.library.ucf.edu/facultybib2000/7070

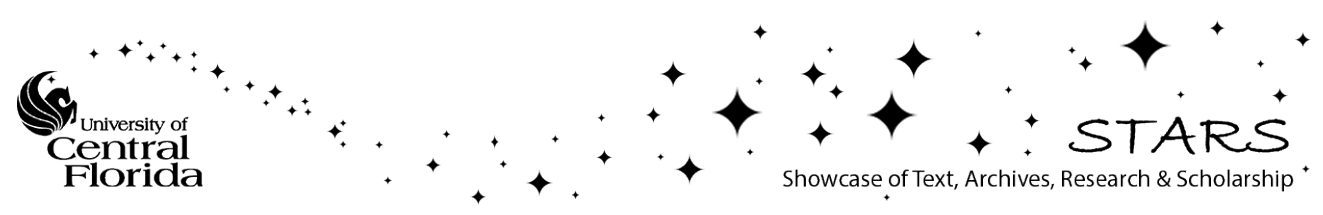




\title{
High-energy two-electron capture with emission of a single photon
}

\author{
E. G. Drukarev, ${ }^{1,2}$ A. I. Mikhailov, ${ }^{1,2}$ I. A. Mikhailov, ${ }^{1,3}$ and W. Scheid ${ }^{2}$ \\ ${ }^{1}$ Petersburg Nuclear Physics Institute, Gatchina, St. Petersburg 188300, Russia \\ ${ }^{2}$ Justus-Liebig-Universität Giessen, Giessen 35392, Germany \\ ${ }^{3}$ University of Central Florida, Orlando, Florida 32816, USA
}

(Received 5 July 2007; published 6 December 2007)

\begin{abstract}
We investigate the two-electron capture with emission of a single photon to the ground state in the Coulomb field of a heavy nucleus in its collision with a light atom. Describing electron-electron interactions in the bound state perturbatively, we obtained an analytical formula for the high-energy limit of the cross section. In combination with previous results obtained in the same approach we calculated the cross section in a broad interval of energies of the collision. We show that the amplitude of the process at high energy depends on the behavior of the bound state wave function near the triple coalescence point. We analyze the properties of the approximate wave functions which are necessary for the description of the high-energy limit.
\end{abstract}

DOI: 10.1103/PhysRevA.76.062701

PACS number(s): 34.80.Lx, 32.80.Fb, 31.25.-v

\section{INTRODUCTION}

Since the first attempts to detect the double electron capture process followed by the emission of a single photon [1], the process became the subject of further experimental [2],[3] and theoretical [4-7] investigations as well. In this case a heavy nucleus is scattered on a light atom, and the two electrons are captured from the atom to the ground state of the heavy nucleus. The process is followed by emission of the photons. The channel in which the two electrons are captured independently and thus two photons are emitted is the most probable one. However, the correlated electrons can be captured also with the emission of a single photon. Such a process is a challenge for the theoretical views on the chargetransfer reactions and on correlation effects in atomic systems.

The calculations require certain model assumptions. In the pioneering work [4], the process was considered in the rest frame of the heavy nucleus. Interactions of the electrons inside the target were neglected. The authors considered the reaction as the capture of two electrons from the continuum state of the Coulomb field of the heavy nucleus to its ground state, followed by the emission of a single photon. The electrons in the initial state were assumed to move with the same velocities, being equal to that of the light atom in the rest system of the heavy nucleus. Another calculation in the framework of the same model was carried out in [5]. In [6] the authors went beyond this picture, taking into account the internal motion of the electrons inside the light target. The papers [4-6] gave controversial results. This stimulates further investigations.

In the present paper we consider the high-energy nonrelativistic limit (HENL) of the process. Although this limit was among the subjects of earlier theoretical studies [4,6,7], there was no consistent analysis until now. However, in the highenergy limit one can carry out analytical estimations and calculations, and can study the interplay of various mechanisms of the process. One can also find the necessary properties of approximate bound state wave functions which are needed for an adequate description of the essential physics of the HENL. These properties are thus needed for tracing the energy dependence of the process.
We consider the case of a heavy projectile nucleus with the charge $Z \gg 1$ and a light target atom with the nuclear charge $Z_{1} \ll Z$. We consider the process in the rest system of the projectile nucleus. The HENL means that the velocity of the target $v$ in the projectile system is large compared to the velocities of the electron in the ground state of the heavy nucleus, i.e., $v / c \gg \alpha Z$, with $\alpha=1 / 137$ being the fine structure constant. To simplify the calculations we consider the nonrelativistic energies of colliding systems, thus setting $v \ll c$. We assume also $(\alpha Z)^{2} \ll 1$. This enables us to describe the bound electrons by nonrelativistic functions.

We employ the system of units with $\hbar=c=1$. In these units the condition $v / c \gg \alpha Z$ can be written as

$$
\xi \ll 1,
$$

with $\xi=\eta / p$, while $p=m v$, and

$$
\eta=m \alpha Z \text {. }
$$

We shall carry out calculations in the lowest order of the parameter $\xi$. The velocities of the relative motion of the target electrons are of the order of their velocities with respect to the target nucleus $v_{1} \sim \alpha Z_{1}$, leading thus to effects of the order $v_{1} / v \sim m \alpha Z_{1} / p=\xi Z_{1} / Z \ll 1$. They can be neglected in the leading terms. Thus, the initial state electrons can be considered, following [4], as free electrons in the continuum spectrum of the heavy nucleus, having the same asymptotic momentum $p=m v$ and energy $\varepsilon=p^{2} / 2 m$. The process is characterized usually by the kinetic energy "per nucleon" $E_{N}$ $(\mathrm{MeV} / \mathrm{u})$. The corresponding electron kinetic energy is $\varepsilon$ $=E_{N} m / m_{N}$, with $m_{N}$ standing for the nucleon mass.

In our approach the photon energy of the two-electron capture is fixed by the condition

$$
\omega=2 \varepsilon+I-I_{T},
$$

with $I$ and $I_{T}$ being the two-electron binding energies in the heavy ion and in the light target. Condition (1) means that the electron energies $\varepsilon_{j} \gg I$. In this process both electrons should lose their large momenta $p \gg \eta$, transferring the large momentum 


$$
\mathbf{q}=2 \mathbf{p}-\mathbf{k}
$$

$(q=|\mathbf{q}| \gg \eta)$ to the heavy nucleus with $\mathbf{k}$ standing for the photon momentum. Note that $k=|\mathbf{k}|=\omega$ in units employed in the paper. For the nonrelativistic energies $\varepsilon \ll m$ considered in the present paper $k \ll p$ and we shall use $\mathbf{q} \sim 2 \mathbf{p}$ for estimations. Two mechanisms of the process correspond to two different ways for transferring large momentum $\mathbf{q}$ to the nucleus. It can be transferred by continuum or bound electrons with each act of transfer leading to a small factor in the amplitude. In the latter case the electron should approach the nucleus at distances of the order $1 / q \ll \eta^{-1}$, which are much smaller than the size of the Bohr orbits. This leads to the quenching of the contribution. On the other hand, each interaction between the continuum electron and the nucleus provides a small factor $\xi$ [8]. However, the bound electron participates in the process at distances of the order $\eta^{-1}$ from the nucleus, where its density reaches the largest values. As a result, the two mechanisms provide terms of the same order of magnitude with the partial cancellation of the contributions.

First we make some estimations to feel the size of the amplitude, which can be represented as

$$
\begin{aligned}
F & =\left\langle\Psi\left(\mathbf{r}_{1}, \mathbf{r}_{2}\right)\left|e^{i\left(\mathbf{k} \cdot \mathbf{r}_{1}\right)} \gamma_{1}+e^{i\left(\mathbf{k} \cdot \mathbf{r}_{2}\right)} \gamma_{2}\right| \Phi\left(\mathbf{p}_{1}, \mathbf{p}_{2} ; \mathbf{r}_{1}, \mathbf{r}_{2}\right)\right\rangle \\
& =2\left\langle\Psi\left(\mathbf{r}_{1}, \mathbf{r}_{2}\right)\left|e^{i\left(\mathbf{k} \cdot \mathbf{r}_{1}\right)} \gamma\right| \Phi\left(\mathbf{p}_{1}, \mathbf{p}_{2} ; \mathbf{r}_{1}, \mathbf{r}_{2}\right)\right\rangle .
\end{aligned}
$$

Here $r_{1}, r_{2}$ are the distances between nucleus and the electrons, $\Psi$ and $\Phi$ stand for the true (unknown) two-electron wave functions of the ground state and of the continuum state, with asymptotic momenta $\mathbf{p}_{1}=\mathbf{p}_{2}$ (we will not write the arguments $\mathbf{p}_{1,2}$ of the function $\Phi$ in most of the cases). Also in Eq. (5) $\gamma_{j}, j=1,2$ is the operator of interaction between photon and electron. We employ the velocity form $\gamma_{j}=$ $-i\left(\mathbf{e} \cdot \nabla_{j}\right) / m$, with $\mathbf{e}$ standing for the photon polarization vector. The vertex factor $(4 \pi \alpha)^{1 / 2} /(2 \omega)^{1 / 2}$ is included in the cross section. Further we label the electron, which emits the photon by $j=1$, and set $\gamma_{1}=\gamma$. In capture and ionization processes, involving one electron, the photon momentum $k$ is related to the electron momentum $p$ and thus the amplitudes can be expanded in terms of $k / p \sim(\omega / m)^{1 / 2}$. As we shall see below, in our process $k$ is related to the averaged binding momentum $\eta$ in some of the contributions to the amplitude. We shall keep a nonzero value of $k$ for these cases using thus Eq. (5) for the amplitude. In other cases we set $k=0$ assuming thus

$$
F=2\left\langle\Psi\left(\mathbf{r}_{1}, \mathbf{r}_{2}\right)|\gamma| \Phi\left(\mathbf{p}_{1}, \mathbf{p}_{2} ; \mathbf{r}_{1}, \mathbf{r}_{2}\right)\right\rangle .
$$

We describe the initial and final electronic states by perturbative wave functions $\Phi_{p}$ and $\Psi_{p}$ obtained by an iteration of the Lippmann-Schwinger equation (LSE) [9] which can be written as

$$
F=F^{(0)}+G^{(0)} V_{1} F,
$$

for $F=\Psi$ and $F=\Phi$, with $F^{(0)}$ and $G^{(0)}$ being the undisturbed wave function and the Green function, respectively. In the two-electron ion the total interaction is $V=V_{e N}(1)+V_{e N}(2)$ $+V_{e e}(1,2)$, with $V_{e N}$ and $V_{e e}$ standing for interactions between the electrons and the nucleus and between the elec- trons correspondingly. Here and further $(j)$ denotes the variables corresponding to the $j$ th electron. We employ Eq. (7) with $F^{(0)}$ and $G^{(0)}$ being the functions for the systems of two noninteracting electrons in the Coulomb field of the nucleus, while $V_{1}=V_{e e}$. Since $V_{e e} \sim V_{e N} / Z$, only the first iteration of Eq. (7) will be needed for the calculations in the lowest order of a $1 / Z$ expansion. Thus, we use the perturbative wave functions

$$
\Phi_{p}=\Phi_{C}+G_{C}(2 \varepsilon) V_{e e} \Phi_{C}
$$

for the initial state, and

$$
\Psi_{p}=\Psi_{C}+G_{C}\left(2 \varepsilon_{1 s}\right) V_{e e} \Psi_{C}
$$

for the final state. In Eqs. (8) and (9) $G_{C}$ is the Green function [the reduced one in Eq. (9)] of two noninteracting electrons in the Coulomb field of the nucleus, with $2 \varepsilon$ and $2 \varepsilon_{1 s}$ standing for the energies of the two-electron states; $\quad \Psi_{C}\left(\mathbf{r}_{1}, \mathbf{r}_{2}\right)=\psi_{C}\left(\mathbf{r}_{1}\right) \psi_{C}\left(\mathbf{r}_{2}\right), \quad$ and $\quad \Phi_{C}\left(\mathbf{r}_{1}, \mathbf{r}_{2}\right)$ $=\varphi_{C}\left(\mathbf{p}, \mathbf{r}_{1}\right) \varphi_{C}\left(\mathbf{p}, \mathbf{r}_{2}\right)$, where $\psi_{C}$ and $\varphi_{C}$ are the single particle wave functions for the ground and for continuum states in the Coulomb field, respectively. In Eq. (9), $\varepsilon_{1 s}<0$ is the single-particle energy of the $1 s$ electron in the Coulomb field of the heavy nucleus. We shall also present the amplitudes in terms of the single-particle Green functions.

We shall carry out an expansion of the initial state wave functions and the Green functions in powers of the interaction $V_{e N}$ [8]. The lowest terms of this expansions are suppressed, since they require large momentum to be transferred to the nucleus by the bound electron. Thus, the next-toleading orders in the expansion of the initial state wave functions and the Green functions should be included. These expansions manifest themselves as power series in $\xi$. Each order of such series also depends on the ratio $\eta^{2} / p^{2}=\xi^{2}$, requiring an additional expansion to obtain the $\xi$ series for the amplitude.

To estimate the factor connected with the transfer of large momenta to the nucleus one can describe the wave functions by the first terms on the right-hand sides of Eqs. (8) and (9). Replacing the Coulomb functions $\varphi_{C}$ by plane waves

$$
\varphi_{0}(\mathbf{p}, \mathbf{r})=e^{i(\mathbf{p} \cdot \mathbf{r})},
$$

we find the contribution caused by transfer of a large momentum $2 \mathbf{p}$ to the nucleus by the bound electrons. The contribution to the amplitude is $F_{0}=2\left\langle\psi_{C}|\gamma| \varphi_{0}(\mathbf{p})\right\rangle\left\langle\psi_{C} \mid \varphi_{0}(\mathbf{p})\right\rangle$ with each matrix element describing the transfer of a large momentum $p$ to the nucleus by the bound electron. Using $\psi_{C}(r)=N_{C} \exp (-\eta r)$ with $N_{C}=\eta^{3 / 2} / \pi^{1 / 2}$, we obtain

$$
\left\langle\psi_{C} \mid \varphi_{0}(\mathbf{p})\right\rangle=\frac{8 \pi \eta N_{C}}{\left(p^{2}+\eta^{2}\right)^{2}} .
$$

(Note that this is just the single-particle wave function in momentum space.) Thus, transferring the large momentum $p \gg \eta$ to the nucleus results in a factor $\eta N_{C} / p^{4}$. Similar estimation of the matrix element $\left\langle\psi_{C}|\gamma| \varphi_{0}\right\rangle$ provides the contribution of the order $F_{0} \sim \frac{(\mathbf{e} \cdot \mathbf{p})}{m} \frac{\eta^{2} N_{C}^{2}}{p^{8}}$ to the amplitude. Hence such contributions depend on $Z^{p^{8}}$ as $Z^{5}$.

However, this overestimates the magnitude of the amplitude by a large factor of the order of $Z$ since, as we shall see 
below, such contributions cancel. Thus, one should include the interaction between the electrons which is $Z$ times smaller than their interactions with the nucleus. One of the electrons transfers a large momentum $q=2 p$ to the nucleus absorbing also momentum $p$ of another electron. This leads to the estimation

$$
F \sim \frac{\eta^{4} \nu}{m p^{7}}
$$

with

$$
\nu=m \alpha .
$$

Thus, the amplitude depends on $Z$ as $Z^{4}$.

This estimation is obtained by assuming that both electrons approach the nucleus at small distances of the order $1 / p$. However, there is an alternative mechanism in which one of the continuum electrons transfers the large momentum $q=2 p$ being at the distance $1 / 2 p$ from the nucleus, while the electrons exchange the momentum $p$, being at distances of the order $1 / \eta$ from the nucleus, where the electronic density is large. In this case the process can be viewed as consisting of two steps. One often meets such a situation in $2 \rightarrow 3$ and $3 \rightarrow 2$ reactions (in which there are two particles in the initial state and three particles in the final state, and vice versa) in high-energy atomic physics [10]. In the first step the continuum electron scatters on the nucleus, changing its momentum from $\mathbf{p}$ to $-\mathbf{p}$. In the second step the system of two electrons with momenta $\mathbf{p}$ and $-\mathbf{p}$ is captured by the nucleus with the emission of a single photon. The latter process does not require large momentum to be transferred to the nucleus. This corresponds to the much studied quasifree mechanism in the inverse process of the double photoionization [11]. The amplitude corresponding to this mechanism is thus presented in terms of those of electron-nucleus scattering and of the double photoionization. The former drops as $p^{-2}$, being thus enhanced compared to that describing transfer of a large momentum by the bound electron. However, the amplitude of double photoionization is still proportional to $p^{-4}$. It contains additional small factors since it is forbidden in the dipole approximation [11]. The two-step mechanism leads to the dependence of the amplitude on the additional parameter $\omega / \eta$ (the ratio of the photon momentum to that of the bound electron). Finally, the estimation provided by Eq. (12) is true for $\omega \ll \eta$. For $\omega \gg \eta$ the amplitude obtains an additional factor of the order $\eta / \omega$ unless the angle between the directions of the photon and electron momenta is close to $\pi / 2$. Thus, for this case we find $F \sim \eta^{5} \nu / p^{9}$.

We also carried out the calculation in which initial state interactions are treated perturbatively, while the final state is assumed to be described by the exact wave function (solution of the Schrödinger equation) $\Psi$. We show that the amplitude contains the contributions which depend on the properties of the bound state in terms of the value of the wave function at the origin $\Psi\left(r_{1}=r_{2}=0\right)$. There are also contributions, which depend on the behavior of the wave function near the triple coalescence point $r_{1}=r_{2}=0$. Such contributions cannot be, however expressed in terms of a Taylor expansion for the wave function, since the wave function is singular at the triple coalescence point, with its $r$ expansion containing logarithmic terms $[12,13]$. As far as we know, dynamical processes with amplitudes being sensitive to the triple coalescence point have not been considered until now.

Proceeding in terms of the exact wave function $\Psi$ we find several cancellations between the constituents of the amplitude. These cancellations are based on the local properties of the solutions of Schrödinger equation, known as Kato cusp conditions [14]. The most important one is the cancellation of the terms proportional to $Z^{5}$, which is based on the first Kato condition. Such cancellations take place for the exact wave function, but do not necessary occur for the approximate wave function employed in the calculations. In the present paper we discuss also necessary properties of the approximate wave functions which are needed for the proper description of the process.

We obtained equations for the angular distribution and the total cross section in the HENL (Born approximation) in the energy interval $I \ll \omega \ll m$. The domination of photon emission in the direction orthogonal to that of the electron momenta increases for $\omega \gtrsim \eta$. At these energies the total cross section obtains an additional small factor at $\omega \gg \eta$. We also analyzed the structure of corrections to the HENL and obtained a simple equation, which presents the cross section for all $\xi \lesssim 1$.

We calculate the amplitude by LSE technique in Sec. II. We analyze the amplitude with the exact function $\Psi$ in Sec. III. In Sec. IV we discuss the necessary properties of approximate functions. In Sec. V we calculate the cross section. We summarize in Sec. VI.

\section{CALCULATION OF THE AMPLITUDE}

\section{A. Expression for the amplitude, mechanisms of the process}

Since we consider the nonrelativistic energies of the incoming electrons the space and spin variables can be separated. The final state of the electrons has a total spin $S=0$, and thus the electron wave functions are space symmetric. Using Eqs. (8) and (9) we present the amplitude as

$$
\begin{gathered}
F=2\left(F^{(0)}+F_{a}+F_{b}\right), \quad F^{(0)}=\left\langle\Psi_{C}|\gamma| \Phi_{C}\right\rangle, \\
F_{a}=\left\langle\Psi_{C} V_{e e} G_{C}|\gamma(\mathbf{k})| \Phi_{C}\right\rangle, \quad F_{b}=\left\langle\Psi_{C}|\gamma(\mathbf{k})| G_{C} V_{e e} \Phi_{C}\right\rangle,
\end{gathered}
$$

with the energies of the propagators shown in Eqs. (8) and (9). Here we define $\gamma(\mathbf{k})=\gamma e^{i\left(\mathbf{k} \cdot \mathbf{r}_{1}\right)}$. We find immediately

$$
F^{(0)}=\left\langle\psi_{C}|\gamma| \varphi_{C}\right\rangle\left\langle\psi_{C} \mid \varphi_{C}\right\rangle=0,
$$

vanishing due to the orthogonality of the states $\psi_{C}$ and $\varphi_{C}$. We mentioned this cancellation in the Introduction.

The amplitudes $F_{a}$ and $F_{b}$ are illustrated by the Feynman diagrams of Fig. 1.

Now, following [15] we present Eq. (14) in terms of the single-particle Coulomb Green functions. Presenting

$$
G_{C}(E)=[E-H(1,2)]^{-1},
$$

with $H(1,2)=H(1)+H(2)$ standing for the Hamiltonian of two noninteracting electrons in the Coulomb field of the nucleus, we can write 

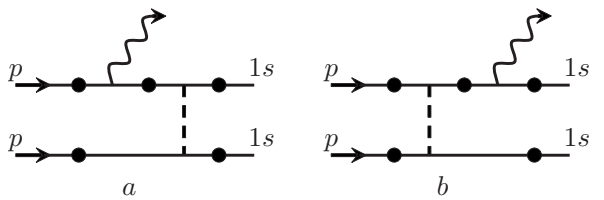

FIG. 1. Feynman diagrams corresponding to the amplitudes $F_{a}$ (a) and $F_{b}$ (b) determined by Eq. (14). The solid lines stand for the electrons, wavy line denotes the photon. The dashed line is for the Coulomb interaction between the electrons. The dark circles show that the electrons are moving in the Coulomb field of the nucleus.

$$
G_{C}(E) \varphi_{C}(2)=\left[E-\varepsilon_{2}-H(1)\right]^{-1} \varphi_{C}(2),
$$

for the amplitude $F_{a}$, and

$$
\psi_{C}(2) G_{C}(E)=\psi_{C}(2)\left[E-\varepsilon_{2}-H(1)\right]^{-1},
$$

for the amplitude $F_{b}$, with $\varepsilon_{2}$ being the eigenvalues of the single-particle wave equations with the Hamiltonian $H(2)$, i.e., $\varepsilon_{2}=\varepsilon$ for Eq. (17) and $\varepsilon_{2}=\varepsilon_{1 s}$ for Eq. (18). Since the first factor on the right-hand side (RHS) of Eq. (17) and the second factor on the RHS of Eq. (18) are just the single-particle Coulomb Green functions

$$
g_{C}(1 ; \epsilon)=[\epsilon-H(1)]^{-1},
$$

we can write

$$
\begin{gathered}
F_{a}=\left\langle\Psi_{C}(1,2) V_{e e} g_{C}\left(1 ; \varepsilon_{a}\right)|\gamma(\mathbf{k})| \Phi_{C}(1,2)\right\rangle, \\
F_{b}=\left\langle\psi_{C}(1,2)|\gamma(\mathbf{k})| g_{C}\left(1 ; \varepsilon_{b}\right) V_{e e} \Phi_{C}(1,2)\right\rangle,
\end{gathered}
$$

with $\varepsilon_{a}=2 \varepsilon_{1 s}-\varepsilon, \varepsilon_{b}=2 \varepsilon-\varepsilon_{1 s}$.

Since the energies of the incoming electrons are large, we can expand the wave functions as well as the Green functions in powers of the $e N$ interaction. As it was explained in the Introduction, the two lowest terms should be included to obtain the lowest term for the expansion of the amplitude in powers of $\xi$,

$$
\begin{gathered}
g_{C}(1 ; \epsilon)=g(1 ; \epsilon)+g(1 ; \epsilon) V_{e N}(1) g(1, \epsilon), \\
\varphi_{C}(i)=\varphi_{0}(i)+g(i ; \epsilon) V_{e N}(i) \varphi_{0}(i),
\end{gathered}
$$

with $i=1,2, \varphi_{0}(i) \varphi_{0}\left(\mathbf{p}_{i}, \mathbf{r}_{i}\right)=e^{i\left(\mathbf{p}_{i} \cdot \mathbf{r}_{i}\right)}$, while $g$ stands for the Green function of free motion. In this approximation we can set also

$$
\varepsilon_{a}=-\varepsilon, \quad \varepsilon_{b}=2 \varepsilon .
$$

Now we can present

$$
\begin{gathered}
F^{(0)}=F_{0}^{(0)}+F_{1}^{(0)}, \quad F_{a}=F_{a 1}+F_{a 2}+F_{a 3}, \\
F_{b}=F_{b 1}+F_{b 2}+F_{b 3},
\end{gathered}
$$

with

$$
\begin{gathered}
F_{0}^{(0)}=\left\langle\Psi_{C}|\gamma| \Phi_{0}\right\rangle, \quad F_{a 1}=\left\langle\Psi_{C} V_{e e} g\left(1 ; \varepsilon_{a}\right)|\gamma| \Phi_{0}\right\rangle, \\
F_{a 2}=\left\langle\Psi_{C} V_{e e} g\left(1 ; \varepsilon_{a}\right) V_{e N}(1) g\left(1 ; \varepsilon_{a}\right)|\gamma| \Phi_{0}\right\rangle,
\end{gathered}
$$
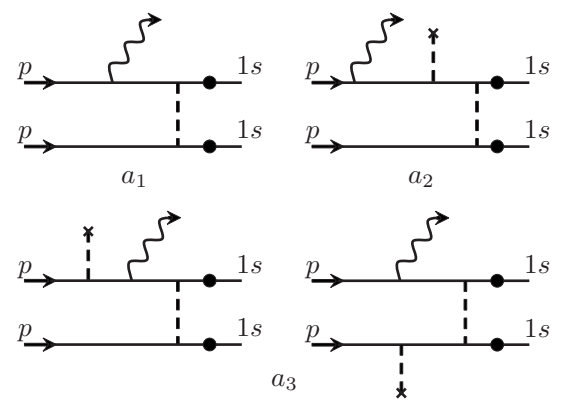

FIG. 2. Feynman diagrams corresponding to the amplitude $F_{a}$ determined by Eq. (24). The labels of the figures correspond to the lower indices of the amplitudes. The dashed line with the cross denotes the Coulomb field of the nucleus. Other notations are the same as in Fig. 1.

$$
\begin{aligned}
F_{a 3}= & \left\langle\Psi_{C} V_{e e} g\left(1 ; \varepsilon_{a}\right)|\gamma(\mathbf{k})| g(1 ; \varepsilon) V_{e N}(1) \Phi_{0}\right\rangle \\
& +\left\langle\Psi_{C} V_{e e} g\left(1 ; \varepsilon_{a}\right)|\gamma(\mathbf{k})| g(2 ; \varepsilon) V_{e N}(2) \Phi_{0}\right\rangle
\end{aligned}
$$

and

$$
\begin{gathered}
F_{1}^{(0)}=\left\langle\Psi_{C}|\gamma| \Phi_{1}\right\rangle, \quad F_{b 1}=\left\langle\Psi_{C}|\gamma| g\left(1 ; \varepsilon_{b}\right) V_{e e} \Phi_{0}\right\rangle, \\
F_{b 2}=\left\langle\Psi_{C}|\gamma| g\left(1 ; \varepsilon_{b}\right) V_{e N}(1) g\left(1 ; \varepsilon_{b}\right) V_{e e} \Phi_{0}\right\rangle, \\
F_{b 3}=\left\langle\Psi_{C}|\gamma(\mathbf{k})| g\left(1 ; \varepsilon_{b}\right) V_{e e} g(1 ; \varepsilon) V_{e N}(1) \Phi_{0}\right\rangle \\
+\left\langle\Psi_{C}|\gamma(\mathbf{k})| g\left(1 ; \varepsilon_{b}\right) V_{e e} g(2 ; \varepsilon) V_{e N}(2) \Phi_{0}\right\rangle
\end{gathered}
$$

with $\Phi_{0}=\varphi_{0}(1) \varphi_{0}(2), \Phi_{1}=g(2 ; \varepsilon) V_{e N}(2) \Phi_{0}$. As we shall see further, the finite values of $\mathbf{k}$ will be important only for the amplitudes $F_{a 3}$ and $F_{b 3}$. The contributions to the amplitude determined by Eqs. (24) and (25) are illustrated by the Feynman diagrams of Figs. 2 and 3 correspondingly.

In the lowest order of expansion in powers of $\xi$ the cancellation expressed by Eq. (15) manifests itself as the cancellation of the amplitudes $F_{0}$ and $F_{1}$. Note that in the former one the second electron is described by the plane wave $\varphi_{0}$, while in the latter one it is described by the lowest order Coulomb correction $\varphi_{1}$. Hence,

$$
F_{0}^{(0)}+F_{1}^{(0)}=\left\langle\psi_{C}|\gamma| \varphi_{0}\right\rangle\left(\left\langle\psi_{C} \mid \varphi_{0}\right\rangle+\left\langle\psi_{C} \mid \varphi_{1}\right\rangle\right)
$$

Note that the corresponding correction to the wave function of the first electron contributes only beyond the HENL due to the factor $\gamma$. Since $V_{e N} \sim Z V_{e e}$ each of these two terms is $Z$
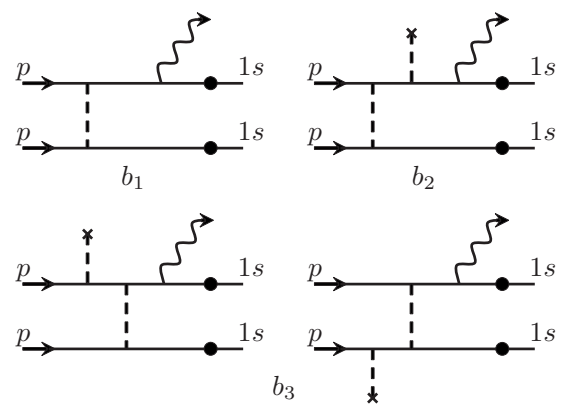

FIG. 3. Feynman diagrams corresponding to the amplitude $F_{b}$, determined by Eq. (25). Notations are the same as in Fig. 2. 
times larger than the other contributions to the amplitude. Employing the expression for the lowest order Coulomb correction to the plane wave

$$
\varphi_{1}(\mathbf{p}, \mathbf{r})=-\int \frac{d^{3} f}{(2 \pi)^{3}} \frac{8 \pi \eta}{p^{2}-f^{2}} \frac{e^{i(\mathbf{f} \cdot \mathbf{r})}}{(\mathbf{p}-\mathbf{f})^{2}},
$$

we find that $\left\langle\psi_{C} \mid \varphi_{0}\right\rangle+\left\langle\psi_{C} \mid \varphi_{1}\right\rangle=0$ in the lowest order of $p^{-1}$ expansion, and thus

$$
F_{0}^{(0)}+F_{1}^{(0)}=0 .
$$

Note that the matrix element $\left\langle\psi_{C} \mid \varphi_{0}\right\rangle$ [Eq. (11)] describes the transfer of the large momentum $p$ to the nucleus by the bound electron, with the distance between them being of the order $1 / p$. On the other hand, the matrix element $\left\langle\psi_{C} \mid \varphi_{1}\right\rangle$ is determined by $r \sim \eta^{-1}, f \sim \eta$. Thus the bound electron remains at distances of the order of the size of the ion, while the continuum electron approaches the nucleus, transferring the large momentum $\sim p$. Interplay of these mechanisms manifests itself in other contributions to the amplitude.

It is instructive to present the amplitude also in another way

$$
\begin{gathered}
F=2\left(F^{(1)}+F^{(2)}\right), \quad F^{(1)}=F_{a 1}+F_{b 1}+F_{a 2}+F_{b 2}, \\
F^{(2)}=F_{a 3}+F_{b 3} .
\end{gathered}
$$

The amplitude $F^{(1)}$ describes the mechanism of the process in which both electrons approach the nucleus on small distances of the order $1 / p$. Large momentum is transferred to the nucleus by one of the electrons and also large momentum is exchanged between the electrons. This is a unique process in which one cannot single out intermediate steps.

The mechanism described by the amplitude $F^{(2)}$ is quite different. One can write for the Green function of free motion

$$
g(\epsilon)=\int \frac{d^{3} p^{\prime}}{(2 \pi)^{3}} \frac{\left|\varphi_{0}\left(\mathbf{p}^{\prime}\right)\right\rangle\left\langle\varphi_{0}\left(\mathbf{p}^{\prime}\right)\right|}{\epsilon-\frac{p^{\prime 2}}{2 m}} .
$$

Using this presentation for the functions $g(1 ; \varepsilon)$ and $g(2 ; \varepsilon)$ in expressions (24) and (25) for the amplitudes $F_{a 3}$ and $F_{b 3}$ we can write

$$
F^{(2)}=\int \frac{d^{3} p^{\prime}}{(2 \pi)^{3}} \frac{F_{\gamma}\left(\mathbf{k}, \mathbf{p}, \mathbf{p}^{\prime}\right)\left\langle\varphi_{0}\left(\mathbf{p}^{\prime}\right)\left|V_{e N}\right| \varphi_{0}(\mathbf{p})\right\rangle}{\epsilon-\frac{p^{\prime 2}}{2 m}} .
$$

Here,

$$
\begin{aligned}
F_{\gamma}\left(\mathbf{k}, \mathbf{p}, \mathbf{p}^{\prime}\right)= & \left\langle\Psi_{C} V_{e e} g\left(1 ; \varepsilon_{a}\right)|\gamma(\mathbf{k})| \Phi_{0}\left(\mathbf{p}, \mathbf{p}^{\prime}\right)\right\rangle \\
& +\left\langle\Psi_{C}|\gamma(\mathbf{k})| g\left(1 ; \varepsilon_{b}\right) V_{e e} \Phi_{0}\left(\mathbf{p}, \mathbf{p}^{\prime}\right)\right\rangle+\left(\mathbf{p} \leftrightarrow \mathbf{p}^{\prime}\right),
\end{aligned}
$$

with

$$
\Phi_{0}\left(\mathbf{p}, \mathbf{p}^{\prime}\right)=\varphi_{0}\left(\mathbf{p}, \mathbf{r}_{1}\right) \varphi_{0}\left(\mathbf{p}^{\prime}, \mathbf{r}_{2}\right),
$$

describing the radiative capture of two free electrons with momenta $\mathbf{p}, \mathbf{p}^{\prime} . F_{\gamma}$ is known to be peaked at $\mathbf{p}+\mathbf{p}^{\prime}=0$, corresponding to a small momentum transferred to the nucleus [11]. Thus, in the amplitude $F_{\gamma}$ large momentum $p$ is ex- changed between the electrons which thus approach each other at distances of the order $1 / p$. However, this takes place at large distances $1 / \eta$ from the nucleus, where the electron density is large.

Hence the integral on the RHS of Eq. (30) is dominated by $\mathbf{p}^{\prime}$ close to $-\mathbf{p}$, i.e., by $\mathbf{p}^{\prime}=-\mathbf{p}+\mathbf{f}$ with $f \ll p$. Presenting $F_{\gamma}\left(\mathbf{k}, \mathbf{p}, \mathbf{p}^{\prime}\right)=\widetilde{F}_{\gamma}(\mathbf{k}, \mathbf{p}, \mathbf{f})$ we can write Eq. (30) as

$$
F^{(2)}=\int \frac{d^{3} f}{(2 \pi)^{3}} \widetilde{F}_{\gamma}(\mathbf{k}, \mathbf{p}, \mathbf{f}) \frac{2 m}{2(\mathbf{p} \cdot \mathbf{f})-f^{2}} \frac{-4 \pi \alpha Z}{q^{2}},
$$

with the last factor corresponding to the second one of the numerator on the RHS of Eq. (30). Hence, the process can be viewed as the electron-nucleus scattering in which the electron changes the direction of its momentum to the opposite one, expressed by the last factor on the RHS of Eq. (33), followed by radiative capture of both electrons with emission of a single photon. The former process takes place at the distances of the order $1 / p$ from the nucleus, the latter one takes place at large distances $1 / \eta$ from the nucleus. The second factor of the integrand is of the order $\eta^{-1} \mathrm{~m} / p$. It is just the time during which the electron with velocity $\mathrm{p} / \mathrm{m}$ passes the distance $1 / \eta$ from the nucleus, i.e., this is the time interval between the two steps of the process. Note that the amplitude $\widetilde{F}_{\gamma}$ depends on $f$ in terms of the ratio $f / p \ll 1$ and of the ratio of $f / \eta_{b} \sim 1$, with $\eta_{b}$ standing for the characteristic momentum of the bound state. Due to certain internal cancellations $[11,16]$, the leading terms of the expansion of $\widetilde{F}_{\gamma}$ in powers of $k / p$ and $f / p$ vanish, and one should include the next terms of the corresponding expansions. More detailed description of the two-step mechanism will be presented in Sec. II C.

\section{B. One-step mechanism}

In this section we neglect the photon momentum, using Eq. (6) for the amplitude, setting thus $\gamma(\mathbf{k})=\gamma(0)=\gamma$. We start with the contribution of the electron interactions in the final state provided by the amplitude $F_{a 1}$ and illustrated by the Feynman diagram of Fig. 2.

Using Eq. (29) for the function $g\left(1, \varepsilon_{a}\right)$, we obtain

$$
F_{a 1}=\int \frac{d^{3} p^{\prime}}{(2 \pi)^{3}} \frac{\left\langle\Psi_{C}\left|V_{e e}\right| \Phi_{0}\left(\mathbf{p}^{\prime}, \mathbf{p}\right)\right\rangle\left\langle\varphi_{0}\left(\mathbf{p}^{\prime}\right)|\gamma| \varphi_{0}(\mathbf{p})\right\rangle}{-\varepsilon-p^{\prime 2} / 2 m} .
$$

Calculating

$$
\left\langle\varphi_{0}\left(\mathbf{p}^{\prime}\right)|\gamma| \varphi_{0}(\mathbf{p})\right\rangle=\frac{(\mathbf{e} \cdot \mathbf{p})}{m}(2 \pi)^{3} \delta\left(\mathbf{p}^{\prime}-\mathbf{p}\right),
$$

and presenting

$$
V_{e e}=\frac{\alpha}{\left|\mathbf{r}_{1}-\mathbf{r}_{2}\right|}=\int \frac{d^{3} s}{(2 \pi)^{3}} \frac{4 \pi \alpha e^{i\left(\mathbf{s} \cdot \mathbf{r}_{1}\right)-i\left(\mathbf{s} \cdot \mathbf{r}_{2}\right)}}{s^{2}},
$$

we obtain 


$$
\begin{aligned}
F_{a 1}= & -\frac{(\mathbf{e} \cdot \mathbf{p})}{p^{2}} \int \frac{d^{3} s}{(2 \pi)^{3}} d^{3} r_{1} d^{3} r_{2} \Psi_{C}\left(\mathbf{r}_{1}, \mathbf{r}_{2}\right) \\
& \times \frac{4 \pi \alpha}{s^{2}} e^{i(\mathbf{p}+\mathbf{s}) \cdot \mathbf{r}_{1}} e^{i(\mathbf{p}-\mathbf{s}) \cdot \mathbf{r}_{2}},
\end{aligned}
$$

which can be presented as

$$
F_{a 1}=-\frac{(\mathbf{e} \cdot \mathbf{p})}{p^{2}} \int \frac{d^{3} s}{(2 \pi)^{3}} \frac{4 \pi \alpha}{s^{2}} \Psi_{C F}(\mathbf{p}+\mathbf{s}, \mathbf{p}-\mathbf{s}) .
$$

The function $\Psi_{C F}(\mathbf{a}, \mathbf{b})=\psi_{C F}(\mathbf{a}) \psi_{C F}(\mathbf{b})$ is the Fourier transform of the function $\Psi_{C}$, while the Fourier transforms $\psi_{C F}$ of the single particle Coulomb wave functions for $1 s$ states are actually presented by Eq. (11). One can see that the integral on the RHS of Eq. (38) is determined by the regions $|\mathbf{p}-\mathbf{s}|$ $\sim \eta \ll p$ and $|\mathbf{p}+\mathbf{s}| \sim \eta \ll p$. This means that only one of the bound electron transfers large momentum $\mathbf{q}$ to the nucleus. The other one exchanges momenta of the order $\eta$ with the nucleus, thus remaining on distances of the order of the Bohr orbit. Thus we obtain

$$
F_{a 1}=-\frac{(\mathbf{e} \cdot \mathbf{p})}{p^{4}} 4 \pi \alpha A_{C}(\mathbf{q})
$$

with

$$
\begin{gathered}
A_{C}(\mathbf{q})=\int \frac{d^{3} f}{(2 \pi)^{3}} \Psi_{C F}(\mathbf{q}-\mathbf{f}, \mathbf{f})=\frac{16 \pi \eta N_{C}^{2}}{\left(q^{2}+4 \eta^{2}\right)^{2}}, \\
N_{C}^{2}=\Psi_{C}(0,0)=\left|\psi_{C}(0)\right|^{2}=\frac{\eta^{3}}{\pi} .
\end{gathered}
$$

In the leading terms of the expansion in powers of $p^{-1}$ we can neglect the binding energies in Eq. (3), setting

$$
\varepsilon=\omega / 2, \quad p^{2}=m \omega .
$$

Introducing

$$
\Lambda_{C}=\frac{4 \pi^{2} \alpha \eta N_{C}^{2}}{p^{8}}(\mathbf{e} \cdot \mathbf{p})=\frac{4 \pi \alpha \eta^{4}}{p^{7}}(\mathbf{e} \cdot \mathbf{n}),
$$

with $\mathbf{n}=\mathbf{p} / p$, we obtain

$$
F_{a 1}=-\Lambda_{C} .
$$

Now we calculate the amplitude $F_{b 1}$ which includes the lowest order electron interactions in the initial state. Using Eq. (25) with presentation (29) for the function $g\left(1 ; \varepsilon_{b}\right)$ and inserting $1=\int \frac{d^{3} f}{(2 \pi)^{3}}\left|\varphi_{0}(\mathbf{f})\right\rangle\left\langle\varphi_{0}(\mathbf{f})\right|$, we write

$$
\begin{aligned}
F_{b 1}= & \int \frac{d^{3} p^{\prime}}{(2 \pi)^{3}} \frac{d^{3} f}{(2 \pi)^{3}} \\
& \times \frac{\left\langle\Psi_{C}\left|\gamma_{1}\right| \Phi_{0}\left(\mathbf{p}^{\prime}, \mathbf{f}\right)\right\rangle\left\langle\Phi_{0}\left(\mathbf{p}^{\prime}, \mathbf{f}\right)\left|V_{e e}\right| \Phi_{0}(\mathbf{p}, \mathbf{p})\right\rangle}{2 \varepsilon-p^{\prime 2} / 2 m} .
\end{aligned}
$$

$$
\left\langle\Phi_{0}\left(\mathbf{p}^{\prime}, \mathbf{f}\right)\left|V_{e e}\right| \Phi_{0}(\mathbf{p}, \mathbf{p})\right\rangle=\frac{4 \pi \alpha}{(\mathbf{p}-\mathbf{f})^{2}}(2 \pi)^{3} \delta\left(\mathbf{q}-\mathbf{p}^{\prime}-\mathbf{f}\right) .
$$

Thus,

$$
F_{b 1}=\int \frac{d^{3} f}{(2 \pi)^{3}} \Psi_{C F}(\mathbf{q}-\mathbf{f}, \mathbf{f}) \frac{4 \pi \alpha}{(\mathbf{p}-\mathbf{f})^{2}} \frac{(\mathbf{e} \cdot \mathbf{q})-(\mathbf{e} \cdot \mathbf{f})}{2 \varepsilon-(\mathbf{q}-\mathbf{f})^{2} / 2 m} .
$$

The integral over $\mathbf{f}$ is determined by small values of $f \sim \eta$. Further evaluation provides

$$
F_{b 1}=-\frac{4 \pi \alpha}{p^{4}}(\mathbf{e} \cdot \mathbf{p}) N_{C} \psi_{C F}(\mathbf{q})
$$

i.e.,

$$
F_{b 1}=-\Lambda_{C}
$$

with $\Lambda_{C}$ defined by Eq. (42).

Proceeding in the same way we find

$$
F_{a 2}=-\Lambda_{C}
$$

while $F_{b 2}$ can be shown to be parametrically smaller (see Sec. II C). Hence, we set $F_{b 2}=0$, thus coming to

$$
F^{(1)}=-3 \Lambda_{C} \text {. }
$$

\section{Two-step mechanism}

In the preceding section we could set $\mathbf{k}=0$ in the photon wave function. Now we keep the finite value of the photon momentum k. For the amplitude $F_{a 3}$ which includes electron-electron interactions in the final state we write

$$
F_{a 3}=X_{1}+X_{2}
$$

with $X_{1}$ describing the scattering by the nucleus and emission of the photon by the same electron, while $X_{2}$ corresponds to the case when different electrons undergo these processes. The amplitudes $X_{1}$ and $X_{2}$ are just the first and second terms on the RHS of expression (24) for $F_{a 3}$. These contributions are illustrated by the Feynman diagrams of Fig. 3. Using Eq. (45) we obtain

$$
\begin{aligned}
X_{i}= & \int \frac{d^{3} s}{(2 \pi)^{3}} \frac{d^{3} s_{1}}{(2 \pi)^{3}} \psi_{C F}\left(\mathbf{s}-\mathbf{s}_{1}\right) \psi_{C F}\left(\mathbf{s}_{1}\right) M_{i}\left(\mathbf{p}, \mathbf{s}, \mathbf{s}_{1}\right) \\
& \times g(\varepsilon,-\mathbf{p}+\mathbf{s}+\mathbf{k}) \frac{-4 \pi \alpha Z}{(\mathbf{q}-\mathbf{s})^{2}}
\end{aligned}
$$

for $i=1,2$ with

$$
M_{1}=\frac{(\mathbf{e} \cdot \mathbf{s})-(\mathbf{e} \cdot \mathbf{p})}{m} h_{1}, \quad h_{1}=-\frac{4 \pi \alpha}{\left(\mathbf{p}-\mathbf{s}_{1}\right)^{2}} \frac{1}{\varepsilon+(\mathbf{p}-\mathbf{s})^{2} / 2 m}
$$




$$
M_{2}=\frac{(\mathbf{e} \cdot \mathbf{p})}{m} h_{2}, \quad h_{2}=-\frac{4 \pi \alpha}{\left(\mathbf{p}-\mathbf{s}_{1}-\mathbf{k}\right)^{2}} \frac{1}{\varepsilon+(\mathbf{p}-\mathbf{k})^{2} / 2 m} .
$$

The amplitude $F_{b 3}$ which includes interactions between electrons in the initial state can be presented in a similar way, i.e., $F_{b 3}=Y_{1}+Y_{2}$, with

$$
\begin{aligned}
Y_{i}= & \int \frac{d^{3} s}{(2 \pi)^{3}} \frac{d^{3} s_{1}}{(2 \pi)^{3}} \psi_{C F}\left(\mathbf{s}-\mathbf{s}_{1}\right) \psi_{C F}\left(\mathbf{s}_{1}\right) L_{i}\left(\mathbf{p}, \mathbf{s}, \mathbf{s}_{1}\right) \\
& \times g(\varepsilon,-\mathbf{p}+\mathbf{s}+\mathbf{k}) \frac{-4 \pi \alpha Z}{(\mathbf{q}-\mathbf{s})^{2}}
\end{aligned}
$$

for $i=1,2$ with

$$
\begin{gathered}
L_{1}=\frac{(\mathbf{e} \cdot \mathbf{s})-\left(\mathbf{e} \cdot \mathbf{s}_{1}\right)}{m} t_{1}, \quad t_{1}=\frac{4 \pi \alpha}{\left(\mathbf{p}-\mathbf{s}_{1}\right)^{2}} \frac{1}{\omega-\left(\mathbf{s}-\mathbf{s}_{1}+\mathbf{k}\right)^{2} / 2 m}, \\
L_{2}=\frac{\left(\mathbf{e} \cdot \mathbf{s}_{1}\right)}{m} t_{2}, \quad t_{2}=\frac{4 \pi \alpha}{\left(\mathbf{p}-\mathbf{s}_{1}-\mathbf{k}\right)^{2}} \frac{1}{\omega-\left(\mathbf{s}_{1}+\mathbf{k}\right)^{2} / 2 m} .
\end{gathered}
$$

The integrals over $\mathbf{s}$ and $\mathbf{s}_{1}$ in Eqs. (51) and (54) are dominated by $s, s_{1} \sim \eta \ll p$. Thus, the leading contributions to the functions $M_{i}$ in Eqs. (52) and (53) are those proportional to $(\mathbf{e} \cdot \mathbf{p})$. However, these terms cancel, and thus one should expand the functions $h_{i}\left(\mathbf{p}, \mathbf{s}, \mathbf{s}_{1}, \mathbf{k}\right)$ in powers of $s_{1} / p$, $s / p$, and $k / p$, taking into account linear terms.

Thus, we can present

$$
F_{a 3}=S_{1}+S_{2}+S_{3}
$$

with $S_{1}$ corresponding to $\mathbf{s}_{1}=\mathbf{s}=\mathbf{k}=0$ in the functions $h_{i}, S_{2}$ coming from expansion of $h_{i}$ in powers of $s / p$ and $s_{1} / p$, while $S_{3}$ originates from that in $k / p$. The higher order terms contribute beyond the HENL. Direct calculation provides

$$
S_{i}=32 \pi^{2} \alpha^{2} Z \frac{m}{p^{4} q^{2}} \int \frac{d^{3} s}{(2 \pi)^{3}} A_{C}(\mathbf{s}) \frac{1}{p^{2}-(\mathbf{p}-\mathbf{k}-\mathbf{s})^{2}} Q_{i},
$$

with $A_{C}(\mathbf{q})$ defined by Eq. (40), $i=1,2,3$ and

$$
Q_{1}=(\mathbf{e} \cdot \mathbf{s}), \quad Q_{2}=-\frac{(\mathbf{e} \cdot \mathbf{p})(\mathbf{p} \cdot \mathbf{s})}{p^{2}}, \quad Q_{3}=\frac{3(\mathbf{e} \cdot \mathbf{p})(\mathbf{p} \cdot \mathbf{k})}{p^{2}} .
$$

The functions $L_{i}$ in Eqs. (55) and (56) are proportional to $s$ and $s_{1}$ and thus one can set $\mathbf{s}_{1}=\mathbf{s}=\mathbf{k}=0$ in the functions $t_{i}$. Hence

$$
F_{b 3}=-32 \pi^{2} \alpha^{2} Z \frac{m}{p^{4} q^{2}} \int \frac{d^{3} s}{(2 \pi)^{3}} A_{C}(\mathbf{s}) \frac{(\mathbf{e} \cdot \mathbf{s})}{p^{2}-(\mathbf{p}-\mathbf{k}-\mathbf{s})^{2}} .
$$

One can see that the contribution $S_{1}$ to $F_{a 3}$ is cancelled by the amplitude $F_{b 3}$, i.e.,

$$
S_{1}+F_{b 3}=0 \text {. }
$$

Note that Eq. (54) was transformed to Eq. (60) without touching the function $A_{C}(\mathbf{s})$. Such transformation can be done for the function $\Psi$ with $A_{C}(\mathbf{s})$ replaced by

$$
A(\mathbf{s})=\int \frac{d^{3} s_{1}}{(2 \pi)^{3}} \Psi_{F}\left(\mathbf{s}-\mathbf{s}_{1}, \mathbf{s}_{1}\right)=\int d^{3} r_{1} \Psi\left(\mathbf{r}_{1}, \mathbf{r}_{1}\right) e^{-i\left(\mathbf{s} \cdot \mathbf{r}_{1}\right)}
$$

This will be employed in the next section.

Thus, the two-step amplitude can be written as

$$
F^{(2)}=\int \frac{d^{3} s}{(2 \pi)^{3}} \frac{2 m}{p^{2}-(\mathbf{p}-\mathbf{k}-\mathbf{s})^{2}}\left[B_{0}(\mathbf{s})+B_{1}(\mathbf{s})\right] \frac{-4 \pi \alpha Z}{q^{2}} .
$$

Here,

$$
B_{0}(s)=-A_{C}(\mathbf{s}) \frac{4 \pi \alpha}{p^{4}}(\mathbf{e} \cdot \mathbf{n})(\mathbf{n} \cdot \mathbf{s}), \quad \mathbf{n}=\mathbf{p} / p
$$

and

$$
B_{1}(s)=A_{C}(\mathbf{s}) \frac{4 \pi \alpha}{p^{4}} 3(\mathbf{e} \cdot \mathbf{n})(\mathbf{n} \cdot \mathbf{k})
$$

compose the amplitude for electron capture with momentum $\mathbf{s}$ transferred to the nucleus. Since the last factor on the RHS of Eq. (63) is the amplitude of $e N$ scattering, Eq. (63) corresponds to Eq. (33). Note that the leading terms in the denominator of the first factor of the integrand on the RHS of Eq. (63) cancel, and thus this factor is of the order $1 / p \eta$. Note that such a cancellation does not occur in the similar expression for the amplitudes $F_{b 2}$. Hence, the latter is suppressed by the additional factor of the order $\xi \ll 1$ and thus can be neglected.

Calculating the integrals

$$
\int \frac{d^{3} s}{(2 \pi)^{3}} \frac{2 m A_{C}(\mathbf{s})}{p^{2}-(\mathbf{p}-\mathbf{k}-\mathbf{s})^{2}}=\frac{m N_{C}^{2}}{p} W,
$$

with

$$
W=\frac{1}{(\mathbf{n} \cdot \mathbf{k})+2 i \eta}
$$

and

$$
\int \frac{d^{3} s}{(2 \pi)^{3}} \frac{2 m(\mathbf{s} \cdot \mathbf{n}) A_{C}(s)}{p^{2}-(\mathbf{p}-\mathbf{k}-\mathbf{s})^{2}}=2 i \eta \frac{m N_{C}^{2}}{p} W,
$$

we obtain

$$
F^{(2)}=\Lambda_{C}[-2 i \eta+3(\mathbf{n} \cdot \mathbf{k})] W
$$

For $|(\mathbf{n} \cdot \mathbf{k})| \ll \eta$, e.g., for all $\omega \ll \eta$, we find thus $F^{(2)}=-\Lambda_{C}$.

\section{The total Born approximation amplitude}

Combining Eqs. (50) and (69) we can now write the HENL for the amplitude, defined by Eq. (28), 


$$
F=-8 \Lambda_{C} \frac{2 i \eta}{(\mathbf{k} \cdot \mathbf{n})+2 i \eta}=-\frac{32 \pi \alpha \eta^{4}}{p^{7}} \frac{(\mathbf{e} \cdot \mathbf{n})}{1-i(\mathbf{k} \cdot \mathbf{n}) / 2 \eta}
$$

Thus, the dipole approximation $k=0$ is valid only for $\omega \ll \eta$. In this case the second term in the denominator can be neglected, and the amplitude is real,

$$
F=-8 \Lambda_{C}
$$

For larger $\omega$ both terms in the denominator should be included. If the angle between the directions of the photon and electron momenta is not too close to $\pi / 2$ the amplitude is mostly imaginary for $\omega \gg \eta$. In this limiting case the amplitude is proportional to $Z^{5}$.

\section{AMPLITUDE IN TERMS OF THE EXACT BOUND STATE WAVE FUNCTION}

\section{A. Expressions for the one-step and two-step amplitudes}

In this section we describe the bound state by the exact wave function $\Psi$ (solution of the Schrödinger equation). The initial state will be still described by the perturbative function $\Phi_{p}$, determined by Eq. (8). The amplitude can be presented in the form similar to Eq. (14),

$$
\begin{gathered}
F=2\left(T_{a}+T_{b}\right), \quad T_{a}=\left\langle\Psi|\gamma(\mathbf{k})| \Phi_{C}\right\rangle, \\
T_{b}=\left\langle\Psi|\gamma(\mathbf{k})| G_{C}(2 \varepsilon) V_{e e} \Phi_{C}\right\rangle,
\end{gathered}
$$

with the amplitudes $T_{a, b}$ being the analogs of the amplitudes $F_{a, b}$ of Sec. II.

Let us start with the analysis of the amplitude $T_{a}$. Using expansion (21) we can present $T_{a}=T_{a 0}+T_{a 3}$, with

$$
\begin{gathered}
T_{a 0}=\left\langle\Psi|\gamma| \Phi_{0}\right\rangle, \\
T_{a 3}=\left\langle\Psi|\gamma(\mathbf{k})| g(1 ; \epsilon) V_{e N}(1) \Phi_{0}\right\rangle+\left\langle\Psi|\gamma(\mathbf{k})| g(2 ; \epsilon) V_{e N}(2) \Phi_{0}\right\rangle .
\end{gathered}
$$

(Analogs of the amplitudes $F_{a 1}$ and $F_{a 2}$ are absorbed in $T_{a 0}$.) In the amplitude $T_{a 0}$ the continuum electrons are described by plane waves, while in the amplitude $T_{a 3}$ the lowest order Coulomb correction is included. Using Eq. (29) for the free Green functions $g$, we obtain

$$
T_{a 3}=\int \frac{d^{3} p^{\prime}}{(2 \pi)^{3}} \frac{R\left(\mathbf{p}^{\prime}, \mathbf{p}\right)\left\langle\varphi_{0}\left(\mathbf{p}^{\prime}\right)\left|V_{e N}\right| \varphi_{0}(\mathbf{p})\right\rangle}{\varepsilon-\frac{p^{\prime 2}}{2 m}},
$$

with

$$
\begin{aligned}
R\left(\mathbf{p}^{\prime}, \mathbf{p}\right)= & \left\langle\Psi|\gamma(\mathbf{k})| \varphi_{0}\left(1, \mathbf{p}^{\prime}\right) \varphi_{0}(2, \mathbf{p})\right\rangle \\
& +\left\langle\Psi|\gamma(\mathbf{k})| \varphi_{0}(1, \mathbf{p}) \varphi_{0}\left(2, \mathbf{p}^{\prime}\right)\right\rangle .
\end{aligned}
$$

As we discussed above, the integral over $\mathbf{p}^{\prime}$ is dominated by the regions, in which only one of the bound electrons transfers large momentum to the nucleus. These are the regions $p^{\prime} \ll p$ and $\left|\mathbf{p}+\mathbf{p}^{\prime}\right| \ll p$, with the contributions $T_{a 3}^{(1)}$ and $T_{a 3}^{(2)}$ to the amplitude correspondingly. Thus we can present

$$
T_{a 3}=T_{a 3}^{(1)}+T_{a 3}^{(2)}
$$

with both terms contributing to the two terms on the RHS of the expression (72) for $T_{a 3}$. One can see that $T_{a 3}^{(1)}$ contributes to the one-step mechanism, while $T_{a 3}^{(2)}$ contributes to the twostep mechanism.

The expression for the amplitude $T_{b}$ can be simplified by expanding Eq. (16) for the Green function in powers of $H(2) / E$, which becomes possible for $E \gg I$ since $\Psi \frac{H(2)}{E}$ $\sim \Psi \frac{I}{E} \ll \Psi$. This enables us to write the amplitude $T_{b}$ in a form similar to (25), i.e.,

$$
\begin{gathered}
T_{b}=T_{b 1}+T_{b 2}+T_{b 3}, \\
T_{b 1}=\left\langle\Psi|\gamma| g(1 ; 2 \varepsilon) V_{e e} \Phi_{0}\right\rangle, \\
T_{b 2}=\left\langle\Psi|\gamma| g\left(1 ; \varepsilon_{b}\right) V_{e N}(1) g\left(1 ; \varepsilon_{b}\right) V_{e e} \Phi_{0}\right\rangle, \\
T_{b 3}=\left\langle\Psi|\gamma(\mathbf{k})| g(1 ; 2 \varepsilon) V_{e e} g(1 ; \varepsilon) V_{e N}(1) \Phi_{0}\right\rangle+\langle\Psi| \gamma(\mathbf{k}) \\
\times\left|g(1 ; 2 \varepsilon) V_{e e} g(2 ; \varepsilon) V_{e N}(2) \Phi_{0}\right\rangle .
\end{gathered}
$$

One can see that setting $\Psi=\Psi_{C}$, we would obtain $T_{b i}=F_{b i}$ $(i=1,2,3)$. As we saw in Sec. II, it is important to keep nonzero values of $\mathbf{k}$ in the amplitude $F_{b 3}$. That is why we do not set $\mathbf{k}=0$ in the amplitude $T_{b 3}$. The amplitude $T_{b 2}$ can be neglected since it contributes beyond the HENL, as well as the amplitude $F_{b 2}$ in Sec. II.

Thus, we find expressions for the one-step amplitude

$$
F^{(1)}=T_{a 0}+T_{b 1}+T_{a 3}^{(1)}
$$

and for the two-step amplitude

$$
F^{(2)}=T_{a 3}^{(2)}+T_{b 3} .
$$

\section{B. One-step mechanism}

First we show the cancellation of the terms proportional to $Z^{5}$, which in the perturbative approach is given by Eq. (15). We can present

$$
T_{a 0}=\int d^{3} r_{1} d^{3} r_{2} \Psi\left(\mathbf{r}_{1}, \mathbf{r}_{2}\right) \gamma e^{i\left(\mathbf{p} \cdot \mathbf{r}_{1}\right)+i\left(\mathbf{p} \cdot \mathbf{r}_{2}\right)}
$$

Here $\mathbf{r}_{1}$ denotes the variables of the electron which emits the photon. The integral on the RHS of Eq. (78) is determined by small values of $r_{j} \sim 1 / p$. Keeping $r_{1}$ to be finite we can expand the function $\Psi\left(\mathbf{r}_{1}, \mathbf{r}_{2}\right)$ near the point $r_{2}=0$,

$$
\Psi\left(\mathbf{r}_{1}, \mathbf{r}_{2}\right)=\Psi\left(\mathbf{r}_{1}, 0\right)+\left.\left(\mathbf{r}_{2} \cdot \nabla_{2}\right) \Psi\left(\mathbf{r}_{1}, \mathbf{r}_{2}\right)\right|_{\mathbf{r} 2=0}+\cdots,
$$

with the gradient calculated at $r_{2}=0$, while dots present the nonlinear terms of expansion, not all of them are presented as Taylor series. The wave function can be presented rather as a generalized power series, which includes the logarithmic terms $[12,13]$. Introducing

$$
\widetilde{\Psi}\left(r_{1}, r_{2}, \rho\right)=\Psi\left(\mathbf{r}_{1}, \mathbf{r}_{2}\right)
$$

with $r_{j}=\left|\mathbf{r}_{j}\right|, \boldsymbol{\rho}=\mathbf{r}_{1}-\mathbf{r}_{2}, \rho=|\boldsymbol{\rho}|$ we can write Eq. (79) as 


$$
\Psi\left(\mathbf{r}_{1}, \mathbf{r}_{2}\right)=\left(1+r_{2} \frac{\partial}{\partial r_{2}}-\frac{\left(\mathbf{r}_{1} \cdot \mathbf{r}_{2}\right)}{r_{1}} \frac{\partial}{\partial \rho}\right) \tilde{\Psi}\left(r_{1}, r_{2}, \rho\right)+\cdots .
$$

The linear terms provide (see Appendix A for details)

$$
T_{a 0}^{\operatorname{lin}}=-\frac{(\mathbf{e} \cdot \mathbf{p})}{m} \frac{8 \pi}{p^{4}} \int d^{3} r_{1} \tilde{\Psi}_{r_{2}}^{\prime}\left(r_{1}, 0, r_{1}\right) e^{i\left(\mathbf{p} \cdot \mathbf{r}_{1}\right)}
$$

with $\widetilde{\Psi}_{r_{2}}^{\prime}$ denoting the partial derivative of the function $\widetilde{\Psi}$ with respect to $r_{2}$ at $r_{2}=0$.

Now we calculate the amplitude $T_{a 3}^{(1)}$ which includes interactions of continuum electrons with the nucleus. As we discussed in the preceding section, this corresponds to the contribution of $p^{\prime} \ll p$ on the RHS of Eq. (73). Due to the vertex operator $\gamma$, only the second term on the RHS of Eq. (74) contributes in the leading order of the $\xi$ expansion. Expanding in powers of $p^{\prime} / p$, i.e., keeping $\mathbf{p}^{\prime}$ only in the wave function $\varphi_{0}\left(2, \mathbf{p}^{\prime}\right)$, we find

$$
T_{a 3}^{(1)}=-\frac{8 \pi \eta}{p^{4}} \int d^{3} r_{1} d^{3} r_{2} \frac{d^{3} p^{\prime}}{(2 \pi)^{3}} \Psi\left(\mathbf{r}_{1}, \mathbf{r}_{2}\right) \gamma e^{i\left(\mathbf{p} \cdot \mathbf{r}_{1}\right)+i\left(\mathbf{p}^{\prime} \cdot \mathbf{r}_{2}\right)} .
$$

Since

$$
\int \frac{d^{3} p^{\prime}}{(2 \pi)^{3}} e^{i\left(\mathbf{p}^{\prime} \cdot \mathbf{r}_{2}\right)}=\delta\left(\mathbf{r}_{2}\right)
$$

we find

$$
T_{a 3}^{(1)}=-\frac{8 \pi \eta}{p^{4}}(\mathbf{e} \cdot \mathbf{p}) J(p), \quad J(p)=\int d^{3} r_{1} \widetilde{\Psi}\left(r_{1}, 0, r_{1}\right) e^{i\left(\mathbf{p} \cdot \mathbf{r}_{1}\right)} .
$$

The integrand on the RHS of Eq. (82) for $T_{a 0}^{\text {lin }}$ can be evaluated by using the first Kato cusp condition [14]

$$
\widetilde{\Psi}_{r_{2}}^{\prime}\left(r_{1}, 0, r_{1}\right)=-\eta \widetilde{\Psi}\left(r_{1}, 0, r_{1}\right),
$$

which can be viewed as the cancellation of singularities in the Schrödinger equation for the ground state of a twoelectron ion at $r_{2}=0$. We obtain immediately

$$
T_{a 0}^{\mathrm{lin}}+T_{a 3}^{(1)}=0 .
$$

The leading contributions of the $1 / Z$ expansion of both terms on the left-hand side of Eq. (87) are proportional to $Z^{5}$. This can be obtained by evaluating (see Appendix A)

$$
J(p)=\frac{8 \pi \alpha Z N^{2}}{p^{4}}\left(1-\frac{1}{2 Z}\right),
$$

with

$$
N^{2}=\Psi(0,0)=\widetilde{\Psi}(0,0,0)
$$

Introducing

$$
\Lambda=\frac{4 \pi^{2} \alpha \eta N^{2}}{p^{7}}(\mathbf{e} \cdot \mathbf{n}),
$$

which turns to $\Lambda_{C}$ defined by Eq. (42) if the parameter $N^{2}$ of the exact function $\Psi$ is replaced by its Coulomb value $N_{C}^{2}$, we can write for the leading contribution to $T_{a 3}^{(1)}$,

$$
T_{a 3}^{(1)}=-16 Z \Lambda \text {. }
$$

For the properly normalized wave function one has $N^{2} \sim Z^{3}$, and thus the leading contribution to the amplitude $T_{a 3}^{(1)}$ is indeed proportional to $Z^{5}$.

Note that the cancellation of the terms proportional to $Z^{5}$ (and of some of the terms, proportional to $Z^{4}$ ) was from the linear terms of the expansion of the solution of the Schrödinger equation near the origin. The contributions of the order $Z^{4}$, to the amplitude $T_{a 0}$ come from the higher order terms of expansion in powers of $r\left(r=r_{1}, r_{2}, \rho\right)$. However, these contributions cannot be presented in terms of the higher order derivatives of the function $\Psi$. This happens because the exact wave function $\Psi$ is singular at $r_{1}=r_{2}=0$, and cannot be presented as a Taylor series.

The other contributions to the amplitude can be expressed in terms of the wave function $\Psi$. Considering the contribution caused by the inclusion of the $e e$ interaction between the continuum electrons, we use Eq. (75) and proceed in the same way as in the calculation of the amplitude $F_{b 1}$ in Sec. II. Employing Eq. (45) we can write the following for the corresponding term of the amplitude:

$$
T_{b 1}=-\frac{8 \pi \nu}{p^{4}} \int d^{3} r_{1} d^{3} r_{2} \frac{d^{3} f}{(2 \pi)^{3}} \Psi\left(\mathbf{r}_{1}, \mathbf{r}_{2}\right) \gamma e^{-i\left(\mathbf{q} \cdot \mathbf{r}_{1}\right)+i \mathbf{f} \cdot\left(\mathbf{r}_{1}-\mathbf{r}_{2}\right)},
$$

with $\nu$ defined by Eq. (13). Using Eq. (84) we obtain

$$
T_{b 1}=-\frac{8 \pi \nu}{p^{4}}(\mathbf{e} \cdot \mathbf{q}) J_{1}(q), \quad J_{1}=\int d^{3} r_{1} \Psi\left(\mathbf{r}_{1}, \mathbf{r}_{1}\right) e^{-i\left(\mathbf{q} \cdot \mathbf{r}_{1}\right)} .
$$

One can see that Eqs. (92) and (93) can be obtained from Eqs. (46) and (47) for $F_{b 1}$ by replacing $\Psi_{C}$ with $\Psi$. Thus, we obtain

$$
T_{b 1}=-\Lambda,
$$

with $\Lambda$ defined by Eq. (90).

\section{Two-step mechanism}

The amplitude is given by Eq. (71). As we noted in Sec. II C, the amplitude $T_{b 3}$ which includes interaction between continuum electrons can be written in terms of the exact function $\Psi$,

$$
\begin{aligned}
T_{b 3}= & -32 \pi^{2} \alpha^{2} Z \frac{m}{p^{4} q^{2}} \int d^{3} r_{1} \frac{d^{3} s}{(2 \pi)^{3}} \Psi\left(\mathbf{r}_{1}, \mathbf{r}_{1}\right) e^{-i\left(\mathbf{s} \cdot \mathbf{r}_{1}\right)} \\
& \times \frac{(\mathbf{e} \cdot \mathbf{s})}{p^{2}-(\mathbf{p}-\mathbf{k}-\mathbf{s})^{2}} .
\end{aligned}
$$

The amplitude $T_{a 3}^{(2)}$ which does not include interactions between continuum electrons can be written as 


$$
T_{a 3}^{(2)}=U_{1}+U_{2}+U_{3},
$$

with

$$
U_{i}=-\frac{8 \pi \alpha Z}{q^{2}} \int d^{3} r_{1} d^{3} \rho \frac{d^{3} s}{(2 \pi)^{3}} \Psi\left(\mathbf{r}_{1}, \mathbf{r}_{2}\right) e^{-i\left(\mathbf{s} \cdot \mathbf{r}_{1}\right)-i(\mathbf{p} \cdot \boldsymbol{\rho})} Q_{i},
$$

where $Q_{i}$ is defined by Eq. (59). Setting $\Psi=\Psi_{C}$ one finds $U_{i}=S_{i}$ for $i=1,2,3$ with $S_{i}$ determined by Eq. (58).

In perturbative approach we had the cancellation expressed by Eq. (61). Similar cancellation

$$
U_{1}+T_{b 3}=0
$$

takes place for the exact wave function $\Psi$. To prove the statement we calculate the integral over $\rho$ on the RHS of Eq. (96)

$$
\int d^{3} \rho e^{-i(\mathbf{p} \cdot \boldsymbol{\rho})} \Psi\left(\mathbf{r}_{1}, \mathbf{r}_{2}\right)=-\frac{8 \pi}{p^{4}} \tilde{\Psi}_{\rho}^{\prime}\left(r_{1}, r_{1}, 0\right),
$$

(see Appendix A), with $\widetilde{\Psi}$ defined by Eq. (80). Using the second Kato condition [14]

$$
\widetilde{\Psi}_{\rho}^{\prime}(r, r, 0)=\frac{\nu}{2} \widetilde{\Psi}(r, r, 0), \quad \nu=m \alpha,
$$

we come to Eq. (97). Hence, the cancellations in the amplitude of the two-step mechanism are also based on the properties of the Schrödinger equation.

Thus we must calculate

$$
F^{(2)}=U_{2}+U_{3} \text {. }
$$

Carrying out the calculations, presented in detail in Appendix $\mathrm{B}$ we find

$$
U_{2}=-\Lambda, \quad U_{3}=i \frac{3 \Lambda \kappa}{2 N^{2}}(\mathbf{n} \cdot \mathbf{k})
$$

with $N^{2}$ and $\Lambda$ defined by Eqs. (89) and (90),

$$
\kappa=\int d r \lambda(r) e^{i k_{p} r}, \quad \lambda(r)=\Psi(\mathbf{r}, \mathbf{r}),
$$

while $k_{p}$ denotes the projection of the photon momentum on the direction of the initial electron momentum p. For $\omega \ll \eta$ the contribution $U_{3}$ leads to the small correction of the order $\omega^{2} / \eta^{2}$ to the cross section.

\section{Total amplitude}

Now we can write the total expression for the amplitude in terms of the exact bound state wave function $\Psi$. For $\omega \ll \eta$ it takes the form

$$
F=2\left(\int d^{3} r_{1} d^{3} r_{2} \Psi\left(\mathbf{r}_{1}, \mathbf{r}_{2}\right) \gamma e^{i\left(\mathbf{p} \cdot \mathbf{r}_{1}\right)+i\left(\mathbf{p} \cdot \mathbf{r}_{2}\right)}-16 Z \Lambda-2 \Lambda\right) .
$$

Here, the first term includes all interactions in the initial state. Its leading contribution, which is proportional to $Z^{5}$ is canceled by the second term [see Eq. (87)]. The third term is the sum of the amplitudes $T_{b 1}$ given by Eq. (94) and $U_{2}$ presented by the first equality of Eq. (101).

The first term is determined by behavior of the wave function at small values of $r_{1} \sim r_{2} \sim 1 / p$. However, since the wave function is not analytical at the point $r_{1}=r_{2}=0$, it cannot be expanded in a Taylor series, and thus cannot be presented in terms of local characteristics of the function $\Psi$. The two other terms on the RHS of Eq. (103) are presented in terms of the value of the function $\Psi$ at $r_{1}=r_{2}=0$.

Replacing the exact function $\Psi$ by the perturbative function $\Psi_{p}$ defined by Eq. (9), with the perturbative expansion for the Coulomb Green function (21), we obtain

$$
\int d^{3} r_{1} d^{3} r_{2} \Psi_{p}\left(\mathbf{r}_{1}, \mathbf{r}_{2}\right) \gamma e^{i\left(\mathbf{p} \cdot \mathbf{r}_{1}\right)+i\left(\mathbf{p} \cdot \mathbf{r}_{2}\right)}-16 Z \Lambda_{C}=-2 \Lambda_{C},
$$

in agreement with the results of Sec. II D.

\section{NECESSARY PROPERTIES OF APPROXIMATE FUNCTIONS}

Actual calculations are often carried out in the standard formalism of quantum mechanics. This means that the calculations are carried out by a straightforward application of Eqs. (5) and (6) for all values of the electron energies. The initial and final states are described by approximate wave functions $\Phi_{a}$ and $\Psi_{a}$. The approximate functions are usually either given numerically or are determined by certain explicit expressions.

In order to be able to reproduce the HENL of the considered process the approximate functions should have certain properties of the exact wave functions, i.e., of solutions of the Schrödinger equation. Start with the initial state wave functions $\Phi_{a}$. As we have seen in Sec. II, describing the initial state by plane waves, we overestimate the value of the amplitude by a large factor $Z \gg 1$. The spurious term is eliminated by taking into account the interactions between the initial state electrons and the heavy nucleus. Hence, the latter should be included into the initial state wave function. Otherwise, we obtain the wrong $Z$ dependence of the amplitude.

Also, as we have seen in Sec. II, the amplitude $F_{b}$ which includes the electron interactions in the initial state provides contributions of the same order of magnitude as the amplitude $F_{a}$ which includes such interactions in the bound state. Thus, the approximate wave function $\Phi_{a}$ should contain such interactions as well.

Turning to the description of the bound state, note first that the cancellation of the terms of the order $Z^{5}$ in the amplitude takes place due to the first Kato condition (86). Hence the approximate function $\Psi_{a}$ should satisfy Eq. (86). Otherwise, we would obtain a wrong $Z$ dependence of the amplitude. Another cancellation of the contributions, expressed by Eq. (97) takes place due to the second Kato condition (99). Hence, to avoid spurious contributions of the same order as those composing the amplitude, we must require that the function $\Psi_{a}$ satisfies the first and the second Kato conditions. 
The last, but not the least, is the behavior of the wave function near the triple coalescence point $r_{1}=r_{2}=0$. This behavior is much more complicated than that of the standard explicit expressions used for approximate wave functions. Hence, during the computations one should separate the region near the point $r_{1}=r_{2}=0$, and use the $r$ expansion of the exact wave function $[12,13]$, improved to satisfy the Kato conditions [17].

\section{TOTAL CROSS SECTION}

Now we calculate the angular distribution of the photons and the total cross section. We can write [5]

$$
d \sigma=\frac{\alpha \omega}{v} N_{1}^{2}|F|^{2} \frac{d \Omega}{2 \pi} .
$$

Here $v=p / m$ stands for velocity of the incoming electrons, $\Omega$ is the solid angle of the emitted photon. Following [5] we assume that the cross section is determined by the contribution of the $K$-shell electrons of the target. Hence, $N_{1}^{2}$ $=\left(m \alpha Z_{1}\right)^{3} / \pi$ with the charge $Z_{1}$ of the nucleus of the target atom is the density of the target electrons. The amplitude $F$ is given by Eq. (70), and summation over polarization of the emitted photons is assumed. Thus the Born approximation cross section $\sigma^{B}$, i.e., the one calculated in the lowest nonvanishing order of expansion in powers of $\xi$ is

$$
\frac{d \sigma^{B}}{d t}=\sigma_{0} \frac{2^{10} \pi Z_{1}^{3}}{Z^{5}} \xi^{13} \frac{1-t^{2}}{1+\mu^{2} t^{2}} .
$$

Here $\sigma_{0}=\alpha^{3} a_{0}^{2}, a_{0}$ is the Bohr radius, $t=(\mathbf{p} \cdot \mathbf{k}) / p k, \mu$ $=\omega / 2 \eta$.

The angular distribution obtains its largest values at small $t^{2}$. This becomes increasingly true with increasing values of $\omega$. At $\omega \gg \eta, \mu \gg 1$ the total cross section is determined by small values of $t^{2} \sim 1 / \mu^{2}$.

In the whole region $m \gg \omega \gg I$ the cross section can be described by the formula

$$
\sigma^{B}=\sigma_{0} \frac{2^{12} \pi Z_{1}^{3}}{3 Z^{5}} \xi^{13} f(\mu),
$$

with

$$
f(\mu)=\frac{3}{2 \mu^{3}}\left[\left(1+\mu^{2}\right) \arctan \mu-\mu\right] .
$$

One can see that $f(\mu) \rightarrow 1$ for $\omega \ll \eta$, while $f(\mu)=3 \pi / 4 \mu$ $+O\left(\mu^{-2}\right)$ for $\omega \gg \eta$.

Equations (105) and (106) provide the leading terms for expansions of the angular distribution and the cross section in powers of $\xi$ at $\xi \rightarrow 0$. At finite values of $\xi$ these equations overestimate the true values. The amplitude of a process in the Coulomb field of the nucleus connected with transfer of large momentum $p \gg \eta$ to the latter contains a characteristic factor $(-1)^{i \xi}=e^{-\pi \xi}[18,19]$. Also, considering the lowest terms of the interactions between the continuum electrons and the nucleus we employed an additional expansion in powers of $\xi^{2}$, neglecting the average binding momentum $\eta$ with respect to the momenta $p$ or $q$-see, e.g., Eq. (40).
Since the experiments were carried out for $\xi \approx 0.8[1-3]$, it is desirable to have an approximate formula for the cross section in a broad interval of the values of $\xi$. The cross section of the process for $\xi \geq 0.1$ was computated in [5]. Here, we present a formula for the cross section, which has a proper high-energy limit (106), approximating also the cross section $\sigma^{(2 e, \gamma)}$ obtained in [5]. Following the arguments presented in the previous paragraph we write for the cross section

$$
\sigma^{(2 e, \gamma)}(\xi)=\frac{\sigma^{B}(\xi) e^{-2 \pi \xi}}{(1+\xi)^{6}}\left(1+a_{1} \xi+a_{2} \xi^{2}\right)
$$

The coefficients $a_{i}(i=1,2)$ are found by best fitting with the cross section obtained within the perturbative model [5]

$$
a_{1}=-6.0, \quad a_{2}=65,
$$

The relative difference between cross section (108) and that obtained in [5] does not exceed $10 \%$ for $\xi<1$.

Of course, single electron capture with emission of a single photon has the largest cross section $\sigma^{(e, \gamma)}$ among the capture processes. In the experiments [1-3], carried out at fixed values of $E_{N}$, the projectiles which captured two electrons were registered in coincidence with the photons carrying the energy determined by Eq. (3). This enables us to distinguish our process from the single electron capture processes since they correspond to singly charged projectiles and from the double electron capture with emission of two photons, in which the energy determined by Eq. (3) is shared by two photons. The cross section of our process $\sigma^{(2 e, \gamma)}$ [approximated by the cross section $\sigma$ at small values of $\xi$-Eq. (106)] is much smaller than $\sigma^{(e, \gamma)}[20]$. In Table I we present the values of these cross sections and those of the cross section for double electron capture with emission of two photons $\sigma^{(2 e, 2 \gamma)}$ [21] for two values of $Z$ and several values of $\xi$. We show the results for $Z=18$ which were actually employed in experiment [1]. Since at fixed values of kinetic energy per nucleon $E_{N}$ and $\xi$ the cross section $\sigma$ drops with increasing $Z$, we present also the results for a smaller value of $Z=12$. In the case of $Z=92[2,3]$ the cross section $\sigma^{(2 e, \gamma)}$ is much smaller at the same values of $\xi$. The results for $\xi=0.84$ are shown since this value corresponds to the conditions of the experiments [1-3]. One can see that there are more chances to detect the process at small values of $Z$.

\section{SUMMARY}

We considered the nonrelativistic high-energy limit for the double electron capture from a light atom to the $K$ shell of a heavy nucleus, followed by the emission of a single photon. High energy means that in the rest frame of the heavy nucleus momenta of the captured electrons $p$ are much larger than the average momentum $\eta$ of the electrons bound by the heavy nucleus.

We show that one can separate two main mechanisms of the process. In the first one both electrons approach the heavy nucleus at small distances $\sim 1 / p$, which are much smaller than the Bohr radius of the $K$ shell. In the second mechanism one of the continuum electrons scatters backward 
TABLE I. Cross sections of the double electron capture with emission of a single photon $\sigma^{(2 e, \gamma)}$, of the single electron capture with emission of a single photon $\sigma^{(e, \gamma)}$, and of the double electron capture with emission of two photons $\sigma^{(2 e, 2 \gamma)}$. The values of the projectile nuclear charge $Z=18$ and of the target nuclear charge $Z_{1}=6$ as well as that of $\xi=0.84$ correspond to conditions of the experiment [1]. Numbers in parentheses brackets denote powers of 10 .

\begin{tabular}{ccccccccc}
\hline \hline$Z$ & $\xi$ & $E_{N}(\mathrm{MeV} / \mathrm{u})$ & $Z_{1}$ & $\sigma^{(e, \gamma)}(\mathrm{kb})$ & $\sigma^{(2 e, 2 \gamma)}(\mathrm{mb})$ & $\sigma^{(2 e, \gamma)}(\mathrm{mb})$ & $\sigma^{(2 e, \gamma)} / \sigma^{(e, \gamma)}$ & $\sigma^{(2 e, \gamma)} / \sigma^{(2 e, 2 \gamma)}$ \\
\hline \multirow{4}{*}{18} & 0.84 & 11.4 & & 0.36 & 1.5 & 3.2 & $8.9(-6)$ & 2.1 \\
& 0.20 & 646 & 6 & $1.5(-3)$ & $2.6(-5)$ & $1.0(-6)$ & $6.7(-10)$ & $3.8(-2)$ \\
& 0.10 & 804 & & $6.4(-5)$ & $4.7(-8)$ & $1.6(-10)$ & $4.0(-12)$ & $3.4(-3)$ \\
& 0.84 & 5.1 & & 0.36 & 1.5 & 24 & $6.7(-5)$ & 16 \\
12 & 0.20 & 287 & 6 & $1.5(-3)$ & $2.6(-5)$ & $7.6(-6)$ & $5.1(-9)$ & 0.29 \\
& 0.10 & 357 & & $6.4(-5)$ & $4.7(-8)$ & $1.2(-9)$ & $1.9(-11)$ & $2.6(-2)$ \\
\hline \hline
\end{tabular}

on the heavy nucleus and transfers the total momentum of the system $\sim 2 \mathbf{p}$ to it. Then, a further exchange of the large momentum $\mathbf{p}$ occurs between the bound electrons, being on the distances of the order of the size of the $K$ shell. The two mechanisms provide contributions of the same order. The description of the second mechanism requires analysis beyond the dipole approximation for $\omega \gtrsim \eta$.

In our analysis we employ a perturbative treatment of interactions between the continuum electrons and the nucleus and between themselves. We show that the contribution corresponding to $V_{e N}=0$ is additionally suppressed, since it requires a large momentum to be transferred to the nucleus by the bound electron. This contribution is cancelled to a large extent by the inclusion of the next order term of the expansion in $V_{e N}$. The cancellation takes place due to the first Kato cusp condition, being thus a feature of the exact solution for the Schrödinger equation. Calculations with $V_{e N}=0$ would overshoot the value of the amplitude by a factor of $Z \gg 1$. This manifested itself in computations carried out in [6]. There are some other cancellations between the contributions to the amplitude, based on the second Kato condition. We note that similar cancellations took place in the amplitude of the double photoionization [16,22].

We found the expression for the amplitude for the case when the bound state is described by the exact wave function [Eq. (103)]. We can separate two contributions. One of them is determined by the value of the wave function at the origin. The other one depends on behavior of the wave function near the triple coalescence point $r_{1}=r_{2}=0$. Thus we consider the dynamical process requiring the treatment of the triple coalescence singularity, which to the best of our knowledge has not been considered previously in the literature. For $Z=18$ [1] the value $\xi=0.1$ corresponds to the energies $E_{N}$ $\sim 800 \mathrm{MeV} / \mathrm{u}$. Such energies are available for nowadays experimental facilities. Unfortunately the cross sections are too small to be detected.

We have analyzed the properties of approximate functions which are necessary to reproduce the proper high-energy limit. The initial state functions should include interactions with the nucleus. The bound state functions should satisfy the Kato cusp conditions and should be corrected for a proper behavior near the triple coalescence point $[12,13,17]$.

Particular calculations were carried out with a perturbative treatment of interactions between the continuum elec- trons and the nucleus and of the interaction between the electrons. The bound state function was built by using the Lippmann-Schwinger equation technique. We show that the interactions between the electrons are of the same importance in initial and final states. Internal motion of the target electrons was neglected. We obtained the angular distribution and the total cross section in the Born approximation for all $I \ll \omega \ll m$. We investigated the structure of the corrections to the Born approximation and found a simple formula which enables us to calculate the cross section in a broad interval of energies, corresponding to $\xi \lesssim 1$ [Eq. (108)].

\section{ACKNOWLEDGMENTS}

The authors thank P. H. Mokler for useful discussions. The work was partially supported by the DFG Grant No. 436 RUS 113/822/. Two of the authors (E.G.D., A.I.M.) acknowledge hospitality during their visits to Justus-LiebigUniversity of Giessen.

\section{APPENDIX A}

We evaluate the integral over $\mathbf{r}_{2}$ on the RHS of Eq. (78), following Ref. [21]. Using the expansion (81) we obtain

$$
\begin{aligned}
\int d^{3} r_{2} \Psi\left(\mathbf{r}_{1}, \mathbf{r}_{2}\right) e^{i\left(\mathbf{p} \cdot \mathbf{r}_{2}\right)} \\
=\int d^{3} r_{2}\left(1+r_{2} \frac{\partial}{\partial r_{2}}-\frac{\left(\mathbf{r}_{1} \cdot \mathbf{r}_{2}\right)}{r_{1}} \frac{\partial}{\partial \rho}\right) \\
\quad \times \tilde{\Psi}\left(r_{1}, r_{2}, \rho\right) e^{i\left(\mathbf{p} \cdot \mathbf{r}_{2}\right)} e^{-\delta r_{2}}, \quad \delta \rightarrow 0 .
\end{aligned}
$$

Since

$$
\int d^{3} r e^{i(\mathbf{p} \cdot \mathbf{r})} e^{-\delta r}=\frac{8 \pi \delta}{\left(p^{2}+\delta^{2}\right)^{2}},
$$

only the second term in parentheses on the RHS of Eq. (A1) provides a nonzero value

$$
\int d^{3} r_{2} \Psi\left(\mathbf{r}_{1}, \mathbf{r}_{2}\right) e^{i\left(\mathbf{p} \cdot \mathbf{r}_{2}\right)}=-\left.\frac{8 \pi}{p^{4}} \frac{\partial \widetilde{\Psi}\left(r_{1}, r_{2}, r_{1}\right)}{\partial r_{2}}\right|_{r_{2}=0} .
$$


Similar calculations enable us to obtain Eq. (98). To obtain Eq. (88) we use the expansion $[12,13]$

$$
\widetilde{\Psi}\left(r_{1}, r_{2}, \rho\right)=1-\eta r_{1}-\eta r_{2}+\frac{\nu}{2} \rho+\cdots
$$

(with the dots denoting nonlinear terms) and apply it to the function $\widetilde{\Psi}\left(r_{1}, 0, r_{1}\right)$.

\section{APPENDIX B}

Here we calculate the amplitudes $U_{2}$ and $U_{3}$ defined by Eq. (96). Integrating over $\rho$ [see Eq. (98)] and using the first Kato condition expressed by Eq. (86), we obtain

$$
U_{2}=-\frac{8 \pi^{2} \alpha Z \nu}{p^{6}}(\mathbf{e} \cdot \mathbf{n}) V_{2}, \quad U_{3}=\frac{8 \pi^{2} \alpha Z \nu}{p^{6}} 3(\mathbf{e} \cdot \mathbf{n})(\mathbf{n} \cdot \mathbf{k}) V_{3},
$$

with

$$
\begin{aligned}
& V_{2}=\int d^{3} r \frac{d^{3} s}{(2 \pi)^{3}} e^{-i(\mathbf{s} \cdot \mathbf{r})} \frac{\lambda(r)(\mathbf{n} \cdot \mathbf{s})}{p^{2}-(\mathbf{p}-\mathbf{s})^{2}}, \\
& V_{3}=\int d^{3} r \frac{d^{3} s}{(2 \pi)^{3}} e^{-i(\mathbf{s} \cdot \mathbf{r})} \frac{\lambda(r)}{p^{2}-(\mathbf{P}-\mathbf{s})^{2}},
\end{aligned}
$$

with $\lambda(r)=\Psi(\mathbf{r}, \mathbf{r})=\widetilde{\Psi}(r, r, 0)$. In the expression for $V_{3}$ the photon momentum $\mathbf{k}$ is not neglected, $\mathbf{P}=\mathbf{p}-\mathbf{k}$. Following the discussion in the main text, we set $\mathbf{P}=\mathbf{p}$ in the expression for $V_{2}$.

For the calculation of $V_{2}$ we present $\mathbf{s} e^{-i(\mathbf{s} \cdot \mathbf{r})}=i \nabla e^{-i(\mathbf{s} \cdot \mathbf{r})}$ and integrate by parts. We find

$$
V_{2}=\frac{i 8 \pi^{2}}{p} \int d^{3} r(\mathbf{p} \cdot \mathbf{r}) e^{-i(\mathbf{p} \cdot \mathbf{r})+i p r} \frac{\lambda^{\prime}(r)}{r^{2}}, \quad \lambda^{\prime}(r)=\frac{d \lambda(r)}{d r} .
$$

Introducing $z=(\mathbf{p} \cdot \mathbf{r}) / p$ and integrating over $z$ we obtain

$$
V_{2}=-\frac{1}{2 p} \int_{0}^{\infty} d r \lambda^{\prime}(r)=\frac{\lambda(0)}{2 p}=\frac{N^{2}}{2 p},
$$

with $N^{2}$ defined by Eq. (89).

One can see also that

$$
V_{3}=-\frac{1}{4 \pi} \int d^{3} r e^{-i(\mathbf{P} \cdot \mathbf{r})+i p r} \frac{\lambda(r)}{r} .
$$

In the lowest order of expansion in powers of $1 / p$ this yields

$$
V_{3}=\frac{-i}{2 p} \kappa
$$

with $\kappa=\int d r \lambda(r) e^{i k_{p} r}$, where $k_{p}$ denotes the projection of the photon momentum on the direction of initial electron momentum p. Since the integral (B6) is saturated by $r \sim 1 / \eta$, we find $\kappa=\int d r \lambda(r)$ for $\left|k_{p}\right| \ll \eta$ (e.g., for all $\left.\omega \ll \eta\right)$.

Using Eqs. (B4) and (B6) we obtain Eq. (101) for the ingredients of the amplitude $F^{(2)}$.
[1] A. Warczak et al., Nucl. Instrum. Methods Phys. Res. B 98, 303 (1995).

[2] G. Bednarz et al., Phys. Scr., T T92, 429 (2001).

[3] G. Bednarz et al., Nucl. Instrum. Methods Phys. Res. B 205, 573 (2003).

[4] V. L. Yakhontov and M. Ya. Amusia, Phys. Rev. A 55, 1952 (1997).

[5] A. I. Mikhailov, I. A. Mikhailov, A. V. Nefeiodov, G. Plunien, and G. Soff, Phys. Lett. A 328, 350 (2004).

[6] A. B. Voitkiv, B. Najjari, N. Toshima, and J. Ulrich, J. Phys. B 39, 3403 (2006).

[7] J. E. Miraglia and M. S. Gavielle, XVI ICPEAC, Book of Abstracts (Queen's University of Belfast, Brighton, 1987), p. 517.

[8] L. D. Landau and E. M. Lifshitz, Quantum Mechanics: Nonrelativistic Theory (Pergamon, Oxford, 1977).

[9] R. Newton, Scattering Theory of Waves and Particles (McGraw-Hill, New York, 1968).

[10] E. G. Drukarev, V. G. Gorshkov, A. I. Mikhailov, and S. G. Sherman, Phys. Lett. 46, 467 (1974).

[11] M. Ya. Amusia, E. G. Drukarev, V. G. Gorshkov, and M. P. Kazachkov, J. Phys. B 8, 1248 (1975); E. G. Drukarev, Phys. Rev. A 52, 3910 (1995).

[12] J. H. Bartlett, Phys. Rev. 51, 661 (1937); V. A. Fock, Izv.
Akad. Nauk SSSR, Ser. Fiz. 18, 161 (1954); Forh. - K. Nor. Vidensk. Selsk. 31, 138 (1958).

[13] P. C. Abbott and E. N. Maslen, J. Phys. A 20, 2043 (1987); J. E. Gottschalk, P. C. Abbott, and E. N. Maslen, ibid. 20, 2077 (1987); J. E. Gottschalk and E. N. Maslen, ibid. 20, 2781 (1987).

[14] T. Kato, Commun. Pure Appl. Math. 10, 151 (1957).

[15] A. I. Mikhailov, I. A. Mikhailov, A. N. Moskalev, A. V. Nefiodov, G. Plunien, and G. Soff, Phys. Rev. A 69, 032703 (2004).

[16] E. G. Drukarev, N. B. Avdonina, and R. H. Pratt, J. Phys. B 34, 1 (2001); T. Suric, E. G. Drukarev, and R. H. Pratt, Phys. Rev. A 67, 022709 (2003).

[17] C. R. Myers, C. J. Umrigar, J. P. Sethna, and J. D. Morgan III, Phys. Rev. A 44, 5537 (1991).

[18] V. G. Gorshkov, A. I. Mikhailov, and V. S. Polikanov, Nucl. Phys. 55, 273 (1964).

[19] H. Bethe and E. E. Salpeter, Quantum Mechanics of One-and Two-Electron Atoms (Academic, New York, 1957).

[20] J. Eichler, Phys. Rep. 193, 165 (1990).

[21] W. E. Meyerhof, R. Anholt, J. Eichler, H. Gould, Ch. Munger, J. Alonso, R. Thieberger, and H. E. Wegner, Phys. Rev. A 32, 3291 (1985).

[22] T. Aberg, Phys. Rev. A 2, 1726 (1970). 